\title{
Plastische und rekonstruktive Chirurgie der Nase eindrucksvoll dargestellt
}

\begin{abstract}
$\triangle$ ußergewöhnliches erwartet den Rezensenten schon auf den ersten Seiten des Buches: der Herausgeber in Lederkluft mit der Rocklegende Udo Lindenberg bei der Übergabe eines Bildes mit dem Titel „... use all piano keys“, wohl als Metapher für den Anspruch des Buches gedacht, alle Tasten der rhinochirurgischen Klaviatur zu bedienen! Sehr selbstbewusst, in der Tat, aber auch ein wirklich origineller Einstieg für ein medizinisches Fachbuch. Chapeau!

Nun, können Herausgeber und sein "Autorenorchester" diesem anspruchsvollen Ziel gerecht werden? Um es gleich vorwegzunehmen, wesentliche Aspekte der plastischen und rekonstruktiven Chirurgie der Nase werden auf weniger als 300 Seiten mit mehr als 1.000 hervorragenden Abbildungen und Zeichnungen präsentiert.
\end{abstract}

In Sektion 1 bearbeiten die Autoren grundlegende Fragen wie Patientenselektion und psychologische oder ethnische Besonderheiten, aber auch Fragen zu handwerklichem Rüstzeug wie der adäquaten Auswahl von Instrumentarium oder Nahtmaterial bzw. der Wahl des richtigen Gewebeersatzmaterials für die unterschiedlichen Verwendungsbereiche wird ausreichend Platz eingeräumt.

Im zweiten Teil werden diverse Frageund Problemstellungen der plastischen und rekonstruktiven Nasenchirurgie bearbeitet. Die einzelnen Kapitel leben hier in erster Linie von der ausgezeichneten Bilddokumentation, das operative Vorgehen wird dem Betrachter schrittweise an Hand professionell erstellter Zeichnungen nahegebracht. Also keine klassische OP-Lehre, sondern eher ein Bildatlas mit eindrucksvollen Falldarstellungen.

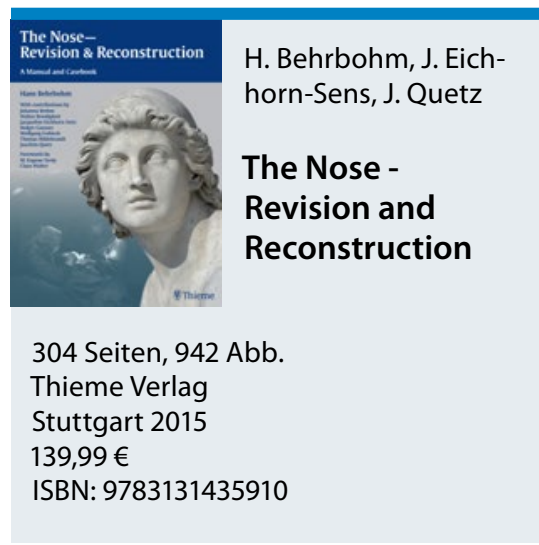

Wer sich mit plastischer und/oder rekonstruktiver Chirurgie der Nase befasst, ist mit diesem Opus bestens bedient, und zwar unabhängig von Ausbildungsstand und Erfahrung.

Dem Herausgeber, der hier mit viel Liebe zum Detail tätig geworden ist, wünsche ich eine weite Verbreitung seines Werkes.

Prof. Gerhard Grevers

\section{Gesammeltes Tinnitus-Fachwissen kompakt präsentiert}

$\mathrm{D}$ ie erste Auflage dieses Buches vor sieben Jahren ist sehr positiv aufgenommen worden, hat jedoch meines Wissens nicht den verdienten Leserkreis gefunden, wohl deshalb, weil mit Tinnitusliteratur der Name Feldmann verbunden ist. Zumindest wer die erste Auflage des Buches „Tinnitus" von Hesse nicht besitzt, jedoch Tinnituspatienten diagnostiziert, behandelt oder begutachtet, für den ist der Erwerb der zweiten überarbeiteten und erweiterten Auflage ein „Muss“. Zehn namhafte deutsche Tinnitusspezialisten haben ihr Fachwissen eingebracht.

Im Kapitel Pathophysiologie hat Hesse die organpathologische Entstehung des Tinnitus, z.B. bei internistischen Erkrankungen oder Kiefergelenk- und HWS-Erkrankungen, erfreulich straff dargestellt, um Platz zu schaffen für die weniger bekannten molekularbiologischen Aspekte (Mazurek, Szczpek) und die Psychopathologie (Schaaf). Das Dia- gnostikkapitel umfasst vorwiegend audiologische Untersuchungsmethoden, aber auch Laboranalysen und Bildgebung sowie psychodiagnostische Verfahren (Schaaf, Goebel). Zur Therapie des akuten und chronischen Tinnitus wird in acht Kapiteln von verschiedenen Autoren (Hesse, Laubert, Kießling, Goebel, Schaaf, Biesinger, Ernst) ausführlich Stellung genommen. Einerseits werden bewährte Verfahren beschrieben wie Hörgeräteanpassung oder Entspannungsverfahren, andererseits werden umstrittene Optionen, z.B. medikamentöse Therapie, Homöopathie, Neuromodulation oder paramedizinische Behandlungsverfahren, kritisch erläutert. Dies betrifft auch Forschungsprogramme, z.B. Gentransfer, Stammzelltherapie und Wachstumsfaktoren bzw. Zytokine. Zur Begutachtung des Tinnitus für die verschiedenen Versicherungsträger und die Beurteilung der Dienstfähigkeit nimmt Michel ausführlich Stellung.

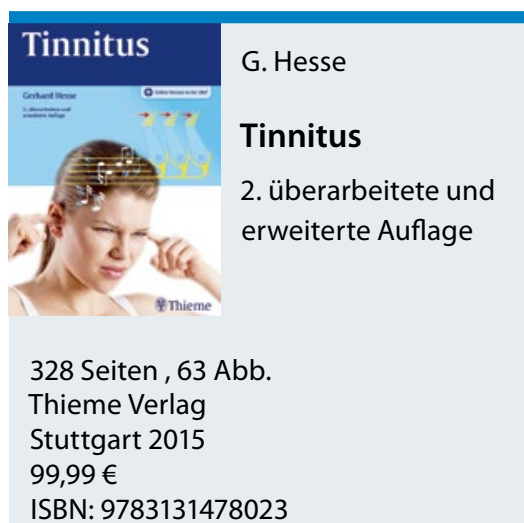

Das Buch ist umfassend und lehrreich und dennoch äußerst übersichtlich und somit einfach und spannend zu lesen. Gelungen ist das Thieme-übliche Layout durch farbliches Hervorheben von Merke-Hinweisen, Fazit, Empfehlungen und Studien. Gerhard Hesse hat es geschafft, trotz der vielen Koautoren ein einheitliches Werk ohne Überschneidungen zu schaffen.

Prof. Karin Schorn 